Conclusion These results suggest the phenotype changes and increase of myocardial capillary pericytes in response to hypertension with diabetes. These changes may contribute to arterialisations of myocardial capillary and pericapillary fibrosis.

\section{e0133 INFLUENCE OF TELMISARTAN ON OXIDATIVE STRESS PARAMETERS IN STREPTOZOTOCIN-INDUCED TYPE 1 DIABETIC RATS}

doi:10.1136/hrt.2010.208967.133

Tang Xiang, Cheng Xunmin, Jiang Shisen, Song Dan. Department of Cardiology, School of Medicine, Nanjing University, Nanjing, China

Objective To investigate the level of oxidative stress in streptozotocin-induced type 1 diabetic rats as well as the intervention effects of telmisartan.

Methods Diabetic rat models were established by intraperitoneal injection of streptozotocin on adult male Wistar rats. The model diabetic rats were randomly divided into two groups of diabetic rats (DM group) and diabetic rats treated with telmisartan ( $\mathrm{T}$ group) that was with eight in each group. There are eight normal rats as the control group (Con group). After 12 weeks, Body weight and heart weight were measured to calculate HW/BW. Lipid Peroxidation (Malondialdehyde, MDA), glutathione (GSH), the activity of superoxide dismutase (SOD) were evaluated by spectrophotometer. Ultra-microstructure of cardiac muscle cell and structure of heart was observed by transmission electron microscope.

Results The blood glucose were found no significant difference between those diabetic rats with and without telmisartan treatment groups at the 7th day after streptozotocin injection and the 12th week, but both higher than that of control group $(p<0.01)$. The level of MDA in DM group was higher (6.92 \pm 0.62 vs $3.66 \pm 0.51, \mathrm{p}<0.01)$ and the level of GSH was lower (7.88 \pm 1.76 vs $11.97 \pm 1.15, p<0.01)$ than those in Con group. The activity of SOD was also lower than those in Con group (155.35 \pm 17.23 vs $219.72 \pm 22.39, \mathrm{p}<0.01)$. Compared with DM group, the level of GSH $(11.22 \pm 1.67$ vs $7.88 \pm 1.76, p<0.01)$ and the activity of SOD $(187.70 \pm 20.59$ vs $155.35 \pm 17.23, \mathrm{p}<0.01)$ increased remarkably in $\mathrm{T}$ group, meanwhile, the level of $\mathrm{MDA}$ in $\mathrm{T}$ group was lower than that of DM group $(4.24 \pm 0.47$ vs $6.92 \pm 0.62, p<0.01)$. Myocardium of DM group was characterised by mitochondrial swelled and crista mitochondriales arranged irregularly or broken even dissolved. In addition, vacuolar degeneration was observed obviously in mitochondria of myocardium. Injury change of ultramicrostructure of myocardium in $\mathrm{T}$ group Lessen obviously than DM group.

Conclusion Telmisartan may inhibit oxidative stress, then improves ultramicrostructure of myocardium in type 1 diabetic rats.

\section{e0134 THE EFFECTS OF TELMISARTAN ON MITOCHONDRIAL MEMBRANE POTENTIAL AND CARDIOMYOCYTE APOPTOSIS IN TYPE 1 DIABETIC RATS}

doi:10.1136/hrt.2010.208967.134

Tang Xiang, Cheng Xunmin, Jiang Shisen, Song Dan. Department of Cardiology, School of Medicine, Nanjing University, Nanjing, China

Objective To investigate the changes of mitochondrial membrane potential and cardiomyocyte apoptosis as welll as the intervention of telmisartan, further to explore whether telmisartan can improve cardiomyocyte apoptosis through the possible pathway of mitochondria correlated in diabetic rats.
Methods Diabetic rat models were established by intraperitoneal injection of streptozotocin on adult male Wistar rats. The model diabetic rats were randomly divided into two groups of diabetic rats (DM group) and diabetic rats treated with telmisartan ( $T$ group) that was with eight in each group. There are eight normal rats as the control group. After 12 weeks, Fluorescent probe DCFH-DA was used to monitored the levels of reactive oxygen species and JC-1 was used to monitored the changes of mitochondrial membrane potential by spectrofluorophotometer. Cardiomyocyte apoptosis was evaluated by TUNEL.

Results The blood glucose were found no significant difference between those diabetic rats with and without telmisartan treatment groups at the 7th day after streptozotocin injection and the 12th week, but both higher than that of control group $(p<0.01)$. Compared with controls, Body weight decreased remarkably in DM group and $\mathrm{T}$ group $(410.63 \pm 11.59$ vs $426.88 \pm 14.32, \mathrm{p}<0.01)$. Cardiomyocyte apoptosis was seen few in control group; compared with control group, cardiomyocyte apoptotic index ascended $(0.35 \pm 0.16$ vs $0.15 \pm 0.08, \mathrm{p}<0.05)$ and mitochondrial membrane potential $(\Delta \Psi \mathrm{m})$ depressed $(9.66 \pm 1.70$ vs $19.88 \pm 1.38, \mathrm{p}<0.01)$ in DM group, but the index of cardiomyocyte apoptosis was lower $(0.17 \pm 0.08$ vs $0.35 \pm 0.16, \mathrm{p}<0.05)$ and transmembrane potential $(\Delta \Psi \mathrm{m})(16.84 \pm 1.84$ vs $9.66 \pm 1.70, \mathrm{p}<0.01)$ in $\mathrm{T}$ group was higher than that of DM group.

Conclusion Telmisartan can promote mitothondrial membrane potential and improve cardiomyocyte apoptosis in type 1 diabetic rats.

\section{e0135 THE EFFECTS OF DIABETIC MELLITUS ON THE OPENING OF MYCARDIAL MITOCHONDRIAL PERMEABILITY TRANSITION PORE IN RATS}

doi:10.1136/hrt.2010.208967.135

Song Dan, Cheng Xunmin, Jiang Shisen, Tang Xiang. Department of Cardiology, Clinic Medical School, Nanjing University/Nanjing General Hospital of Nanjing Military Command, Nanjing, China

Objective To investigate the effects of diabetic mellitus on the opening of mycardial mitochondrial permeability transition pore (MPTP) in rats and explore the role of the opening of MPTP in development of diabetic cardiomyopathy.

Methods 16 male Wistar rats were randomly divided into normal control (NC) group and diabetic mellitus (DM) group. Diabetic mellitus models were established by intraperitoneal injection of streptoaotocin. After 12 weeks, the levels of reactive oxygen species (ROS) and the changes of membrane potential in myocardial mitochondria (MMP) were assayed. Cardiomyocyte apoptosis was evaluted by TUNEL and the level of glutathione (GSH) in serum was measured by spectrophotometer.

Results Compared to the control group, the rate of the ROS generation in the myocardial mitochondria were more significantly increased $(5.10 \% \pm 1.23 \%$ vs $2.42 \% \pm 0.74 \%, p<0.01)$ in DM group but the levels of MMP in the myocardial mitochondria were more significantly decreased $(9.65 \pm 1.69$ vs $19.88 \pm 1.38, p<0.01)$. The apoptotic index was more significantly increased $(0.40 \pm 0.03$ vs $0.26 \pm 0.02, p<0.01$ )and the levels of GSH in serum were more significantly decreased $(7.88 \pm 1.76$ vs $11.97 \pm 1.15, p<0.01)$ in $D M$ group than NC group. It was negative correlation between the rate of the ROS generation and the levels of MMP in the myocardial mitochondria $(\mathrm{p}<0.05)$.

Conclusions Diabetic mellitus may lead the mitochondrial oxidative stress and induce MPTP opening and lead to cell apoptottic consequentely. 